\title{
PARAMETRIZING SMOOTH COMPACTLY SUPPORTED WAVELETS
}

\author{
RAYMOND O. WELLS, JR.
}

\begin{abstract}
In this paper a concrete parameter space for the compactly supported wavelet systems of Daubechies is constructed. For wavelet systems with $N$ (generic) nonvanishing coefficients the parameter space is a closed convex set in $\mathbf{R}^{(N-2) / 2}$, which can be explicitly described in the Fourier transform domain. The moment-free wavelet systems are subsets obtained by the intersection of the parameter space and an affine subspace of $\mathbf{R}^{(N-2) / 2}$.
\end{abstract}

\section{INTRODUCTION}

Compactly supported orthogonal systems of wavelets were introduced recently by Daubechies [1], generalizing a specific example due to Haar [2] known for some time. Daubechies proved the existence of a multidimensional family of such wavelet systems, and, in particular, specific wavelet systems with maximally vanishing moments and with smoothness properties of a very specific type. The wavelets are classified in a rough manner by the number of nonvanishing coefficients in the fundamental difference equation which defines them. This is an even integer $N, N \geq 2$, and, the support of a fundamental scaling function has length $N-1$. As the number of coefficients increases, the support gets larger, and the smoothness is allowed to increase. If a wavelet system is $C^{\infty}$ then there are necessarily an infinite number of coefficients and the support is the entire real axis.

If $N=2$, there is (up to translation), only one wavelet system, the Haar system. If $N=4$, there is a one-parameter family of wavelet systems, and more generally, if $D=(N-2) / 2$, then for arbitrary even $N$, there is a $D$-parameter family of wavelet systems. This last fact is proved in Pollen [4], and we summarize this work in $\S 2$. Pollen gives an explicit parametrization of wavelet systems based on the understanding of the algebraic solution to the constraints which the coefficients of the defining difference equation for a wavelet system must satisfy. This parametrization depends on a specific product which Pollen develops on the abstract set of all wavelet systems, and which enables one to express high order wavelet systems as products of low order ones.

If we let $\mathscr{M}_{N}$ be the set of all solutions of the wavelet coefficient constraints, the moduli space for wavelet systems (see $\S 2$ for details), then $\mathscr{M}_{N}$ has the structure of a compact real algebraic variety in $\mathbf{R}_{N}$ of dimension $D$. More

Received by the editors January 30, 1990 and, in revised form, May 22, 1991.

1980 Mathematics Subject Classification (1985 Revision). Primary 41A58, 42C15.

This material is based upon work supported by AFOSR under grant number 90-0334 which was funded by DARPA. Research also supported by Aware, Inc., and the National Science Foundation. 
specifically it is a torus of dimension $D$ with some subtori collapsed to points, a "pinched torus" of higher dimension. In particular, $\mathscr{M}_{6}$ is a torus $S^{1} \times S^{1}$ with one specific circle $S^{1}$ pinched to a point, and that point is the Haar system.

In this paper we want to present a somewhat different parametrization of wavelet systems, which we call the reduced moduli space $\mathscr{R}_{N}$. The reduced moduli space $\mathscr{R}_{N}$ is a convex compact set contained in $\mathbf{R}^{D}$ which has an interior $\stackrel{\circ}{\mathscr{R}}_{N}$ and, moreover, $\mathscr{R}_{N}$ is the closure of $\stackrel{\circ}{\mathscr{R}}_{N}$. There is an algebraic mapping $\pi: \mathscr{M}_{N} \rightarrow \mathscr{R}_{N}$ which is surjective, and finite-to-one. The fiber of the mapping $\pi$ consists of wavelet systems which have the same smoothness properties, moment vanishing, etc., as the reduced moduli space effectively parametrizes these important quantities, as we shall see.

In $\S 3$ we give the parametrization, depending on two fundamental lemmas from Daubechies' paper [1]. In $\S 4$ we show how the wavelet systems with moments vanishing to a specified order are embedded as affine submanifolds of $\mathscr{R}_{N}$, and in $\S 5$ we explicitly compute extremal points of $\mathscr{R}_{N}$ which are vertices of polyhedra which are suspensions of the one-dimensional reduced moduli space $\mathbf{R}_{\mathbf{4}}=[-16,2]$.

This parametrization has the advantage of global coordinates in $\mathbf{R}^{d}$, explicit smoothness dependencies on the parameters (to be developed in a subsequent paper), and a simple geometry which should facilitate optimization problems. The general goal would be to optimize wavelet systems to be adapted to a specific problem. There are two ways this might happen: (1) choose a wavelet system for an application, optimizing over $\mathscr{R}_{N}$ or (2) dynamically optimizing over $\mathscr{R}_{N}$, modifying the choice of wavelet at successive stages as the application progresses with respect to some parameter (e.g., time). We plan to study examples of both types of behavior in future investigations.

\section{Compactly supported wavelets and the Pollen parametrization}

For each $N, N=2,4,6, \ldots$, let $\mathscr{M}_{N}$ be the set of solutions of the equations:

$$
\begin{aligned}
\sum_{k=0}^{N-1} a_{k} & =2, \\
\sum_{k=0}^{N-1} a_{k} a_{k+2 l} & =2 \delta(l), \quad l=0,1,2, \ldots
\end{aligned}
$$

This is a finite set of algebraic equations in $\mathbf{R}^{N}$.

For each solution $a \in \mathscr{M}_{N}$ of (2.1) and (2.2) we let $\phi$ be a solution of the difference equation

$$
\phi(x)=\sum_{k=0}^{N-1} a_{k} \phi(2 x-k),
$$

which is in $L^{2}(\mathbf{R})$ and has support in the interval [0,N-1]. Daubechies has proved that, (see $[1,3]$ ) for any such $a$, there always exists unique $L^{2}$ solutions of $\left(2.2^{\prime}\right)$. If we let, for such a scaling function $\phi(x)$ satisfying $\left(2.2^{\prime}\right)$,

$$
\psi(x):=\sum_{k=-1}^{N-2} a_{k+1}(-1)^{k} \phi(2 x+k),
$$


and

$$
\phi_{k}(x):=\phi(x-k), \quad \psi_{j k}(x):=2^{j / 2} \psi\left(2^{j} x-k\right),
$$

then $\left\{\phi_{k}, \psi_{j k}\right\}$ is the wavelet system defined by the parameter $a \in \mathscr{M}_{N}$.

Pollen has given a very beautiful description of the solutions to the equations (2.1), (2.2). Each $\mathscr{M}_{N}$ is, by definition, a real-algebraic variety, and we observe, that $\mathscr{M}_{2} \subset \mathscr{M}_{4} \subset \cdots \subset \mathscr{M}_{N}$, since $a=\left(a_{0}, \ldots, a_{L}\right) \in \mathscr{M}_{L}$ implies that $\left(a_{0}, \ldots, a_{L}, 0, \ldots, 0\right) \in M_{L+2 l}$, if we add on $2 l$ zeros to the $L$-type $a$. It is trivial to check that $\mathscr{M}_{2}=\{(1,1)\}=H$ and that this corresponds precisely to the Haar basis (see [1]). Similarly $\mathscr{M}_{4}$ is given as a circle, with a distinguished point $H \in \mathscr{M}_{4}$ being the Haar solution. Daubechies has constructed a specific $N=4$ solution which we call $D_{4}$, which is also a point on $\mathscr{M}_{4}$. Specifically,

$$
D_{4}=\left(\frac{1+\sqrt{3}}{4}, \frac{3+\sqrt{3}}{4}, \frac{3-\sqrt{3}}{4}, \frac{1-\sqrt{3}}{4}\right) \text {. }
$$

The parametrization of $M_{4}$ is given by $a(\theta)$, where $a=\left(a_{0}, a_{1}, a_{2}, a_{3}\right)$ and $a_{j}(\theta)$ are given by

$$
\begin{array}{ll}
a_{0}=\frac{1}{2}(1-\cos \theta+\sin \theta), & a_{1}=\frac{1}{2}(1-\cos \theta-\sin \theta), \\
a_{2}=\frac{1}{2}(1+\cos \theta-\sin \theta), & a_{3}=\frac{1}{2}(1+\cos \theta+\sin \theta) .
\end{array}
$$

We see that if we let $\theta$ take on particular values we obtain

$$
\begin{array}{ll}
a(0)=(0,1,1,0), & a(\pi / 2)=(1,1,0,0), \\
a(\pi)=(1,0,0,1), & a(3 \pi / 2)=(0,0,1,1) .
\end{array}
$$

We can identify $a(0), a(\pi / 2)$, and $a(3 \pi / 2)$ with translates of the Haar solution $H=(1,1,0,0)$, while $a(\pi)$ is a degenerate solution. We see that for $\theta=7 \pi / 6,(2.3)$ yields the smooth Daubechies scaling function $D_{4}$.

In general there is a mapping, due to Pollen (letting $D=(N-2) / 2$ be the dimension of the algebraic variety $\left.\mathscr{M}_{N}\right)$ of the form $\left(S^{1}\right)^{D} \stackrel{\mathscr{P}}{\rightarrow} \mathscr{M}_{N}$, which is the Pollen parametrization of $\mathscr{M}_{N}$. The mapping is generally of maximal rank and surjective, but it degenerates along specific lower dimensional submanifolds of $\left(S^{1}\right)^{D}$, the parameter space. For instance, if we look at $\mathscr{M}_{6}$, and specify $H$ as the Haar solution, then $P^{1}(H)$ is a circle $\gamma$ in $S^{1} \times S^{1}$, and the mapping degenerates on $\gamma$, but is maximal rank elsewhere. In general, the mapping degenerates on lower-dimensional tori contained in $\mathscr{M}_{N}$, as explicitly given by Pollen [4]. The parametrization uses a product structure on $\mathscr{M}_{N}$ which allows one to parametrize the higher-dimensional moduli spaces in terms of lowerdimensional ones. The mapping $\mathscr{P}_{N}$ in higher dimensions can be explicitly given in terms of trigonometric functions just as in (2.3), but we will not be more explicit than we have to at this point, although we later deal with $M_{6}$ in more detail.

\section{THE FOURIER TRANSFORM PARAMETRIZATION}

In this section we want to renormalize the wavelet coefficients by setting

$$
h_{k}:=a_{k} / 2 \text {. }
$$


Then we find that

$$
\sum_{k=0}^{N-1} h_{k}=1, \quad \sum_{k} h_{k} h_{k+2 l}=\frac{1}{2} \delta(l) .
$$

We let $h=\left(h_{0}, \ldots, h_{N-1}\right)$ be a point of the moduli space $\mathscr{M}_{N}$ under this renormalization (change of scale in $\mathbf{R}^{N}$ ). This makes the analysis of the Fourier transform domain much simpler, and will eliminate many factors of powers of 2.

Let now $h \in \mathscr{M}_{N}$, then associated with $h=\left(h_{0}, \ldots, h_{N-1}\right)$ is a trigonometric polynomial

$$
H(\xi):=\sum_{k=0}^{N-1} h_{k} e^{i k \xi}
$$

Since $h \in \mathscr{M}_{N}$, it follows that there is a factorization

$$
H(\xi)=\left(\left(1+e^{i \xi}\right) / 2\right) Q_{0}(\xi)
$$

where $Q_{0}$ is a trigonometric polynomial. This is easy to verify. Namely if we write $Q_{0}=\sum_{k=0}^{N-2} \alpha_{k} e^{i k \xi}$, then (3.1) implies that

$$
\begin{aligned}
h_{0} & =\alpha_{0}, \\
h_{1} & =\alpha_{1}+\alpha_{0}, \\
h_{2} & =\alpha_{2}+\alpha_{1}, \\
\vdots & \\
h_{N-2} & =\alpha_{N-2}+\alpha_{N-1}, \\
h_{N-1} & =\alpha_{N-2} .
\end{aligned}
$$

But then we see that we must have the $h$ 's satisfy one linear condition for these equations to be satisfied. The linear condition is

$$
\sum_{k} h_{2 k}=\sum_{k} h_{2 k+1}
$$

which is satisfied by any point $h \in \mathscr{M}_{N}$. Thus we always have such a factorization such as (3.1).

We now formulate a theorem due to Daubechies [1].

Theorem 3.1. Let $N$ be an even integer $\geq 2$, and let $H(\xi)$ be a trigonometric polynomial of the form $H(\xi)=\sum_{k=0}^{N-1} h_{k} e^{i k \xi}$, and suppose that, for $M a$ nonnegative integer,

$$
H(\xi)=\left(\left(1+e^{i \xi}\right) / 2\right)^{M+1} Q_{M}(\xi)
$$

where

$$
Q_{M}(\xi)=\sum_{k=0}^{N-M-2} \alpha_{k} e^{i k \xi}
$$

then

$$
|H(\xi)|^{2}+|H(\xi+\pi)|^{2}=1
$$

if and only if

$$
\left|Q_{M}(\xi)\right|^{2}=P_{M}\left(\sin ^{2}(\xi / 2)\right)+\sin ^{2 M+2}(\xi / 2) R(\cos \xi),
$$


where

$$
P_{M}(\xi)=\sum_{k=0}^{M}\left(\begin{array}{c}
M+k \\
k
\end{array}\right) y^{k}
$$

and, letting $D=(N-2) / 2, R$ is an odd polynomial of the form

$$
R_{M}(y)=\sum_{k=1}^{D-M} b_{k} y^{2 k-1}
$$

which satisfies

$$
P_{M}(y)+y^{M+1} R_{M}\left(\frac{1}{2}-y\right) \geq 0, \quad \text { for } 0 \leq y \leq 1 .
$$

In the case where $D=M$, then $R_{M}(y)$ is vacuously defined, and we have

$$
\left|Q_{D}(\xi)\right|^{2}=P_{D}\left(\sin ^{2}(\xi / 2)\right) \text {. }
$$

Daubechies [1] has indicated that

$$
|H(\xi)|^{2}+|H(\xi+\pi)|^{2}=1
$$

and

$$
H(\xi)=\left(\left(1+e^{i \xi}\right) / 2\right) Q_{0}(\xi)
$$

is equivalent to $a \in M_{N}$. The quadratic conditions in (3.2) are equivalent to the quadratic conditions in (2.2), and the linear condition in (3.3) are equivalent to the linear conditions in (2.1) (in conjunction with the quadratic conditions), all of which is easy to verify. So the variable coefficients in $Q_{M}$ given in Theorem 3.1 contain the moduli information. Daubechies' theorem, Theorem 3.1, gives a canonical form for the coefficients of $\left|Q_{0}\right|^{2}$.

In the case where $M=0$, we see that $P_{0}(\xi)=1$ and

$$
\left|Q_{0}(\xi)\right|^{2}=1+\left[\sin ^{2}(\xi / 2)\right] R(\cos \xi) .
$$

What is the degree of $R$ ? We see that if we write out $Q_{0}$,

$$
\left|Q_{0}(\xi)\right|^{2}=c_{0}+c_{1} \cos \xi+\cdots+c_{N-2} \cos (N-2) \xi,
$$

where $c_{0}=\alpha_{0}^{2}+\cdots+\alpha_{N-2}^{2}$, etc., and this is a trigonometric polynomial is $\cos \xi$ of order $N-2$. If we write out $\left|Q_{0}(\xi)\right|^{2}$ in terms of the canonical form we see that

$$
\left|Q_{0}(\xi)\right|^{2}=1+\left(\frac{1-\cos \xi}{2}\right)\left(b_{1}(\cos \xi)+\cdots+b_{L}(\cos \xi)^{2 k-1}\right)
$$

which is a trigonometric polynomial in $\cos \xi$ of order $2 L$. Thus we find that $2 L=N-2$, or $L=(N-2) / 2$, which is the dimension of $M_{N}$. The coefficients $b_{i}$ in $R(y)$ are not arbitrary, but must satisfy the following constraint:

$$
P_{M}(y)+y^{M+1} R_{M}\left(\frac{1}{2}-y\right) \geq 0 \quad \text { for } 0 \leq y \leq 1 .
$$

This is a constraint on the coefficients of $R_{M}$. Note that $P_{M}(y)=\sum_{k=0}^{N-1} c_{k} y^{k}$, $c_{k}>0$, is positive for all $y \in[0,1]$.

Noting that $P_{0}(\xi)=1$, we define

$\mathscr{R}_{N}=\left\{\left(b_{1}, b_{2}, \ldots, b_{D}\right) \in \mathbf{R}^{D}: 1+y \sum_{k=0}^{D} b_{k}\left(\frac{1}{2}-y\right)^{2 k-1} \geq 0\right.$, for $\left.0 \leq y \leq 1\right\}$.

This will be the reduced moduli space for the wavelet coefficients. 
Theorem 3.2. There is an algebraic mapping $\pi$

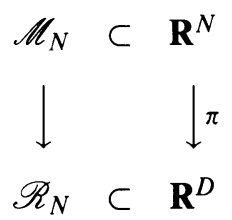

whose restriction to $M_{N}$ is a surjective mapping onto $\mathscr{R}_{N}$.

The mapping is a vector-valued mapping $\pi(a)=\left(b_{1}(a), \ldots, b_{D}(a)\right)$, where each component $b_{j}(a)$ is a homogeneous quadratic polynomial. The generic degree of the mapping is therefore of degree $2^{D}$. This is considering the mapping as a complex-valued algebraic mapping, since each component $b_{j}(a)$ has degree 2 as a mapping, i.e., is a two-to-one generically. Thus we obtain the generic $2^{D}$ mapping as a complex-valued covering mapping $\pi: \mathbf{C}^{N-1} \rightarrow \mathbf{C}^{D}$. What we need to do is investigate the multiplicity of the mapping $\pi: \mathscr{M}_{N} \rightarrow \mathscr{R}_{N}$. In general a real-valued algebraic mapping need not be surjective, in contrast to the complex algebraic situation, where the fundamental theorem of algebra always insures solutions.

Proof. The fundamental idea is the lemma of Riesz (see [4]; cf. [1], used in the proof of Theorem 3.1).

Lemma 3.3. Let $A$ be a trigonometric polynomial with real coefficients containing only cosines,

$$
A(\xi)=\sum_{n=0}^{I} a_{n} \cos (n \xi), \quad a_{n} \in \mathbf{R},
$$

and suppose that $A(\xi)$ is nonnegative, i.e., $A(\xi) \geq 0$ for all $\xi \in \mathbf{R}$. Then there exists a trigonometric polynomial $B(\xi)=\sum_{n=0}^{I} b_{n} e^{i n \xi}$, with real coefficients $b_{n}$ such that $|B(\xi)|^{2}=A(\xi)$. Moreover, there is a unique choice of $B(\xi)$ of the form

$$
B(\xi)=c \prod_{k=1}^{K}\left(e^{i \xi}-r_{j}\right) \prod_{j=1}^{J}\left(e^{2 i \xi}-2 e^{i \xi} \operatorname{Re} z_{j}+\left|z_{j}\right|^{2}\right)
$$

where $c>0, r_{j} \in(-1,1), z_{j} \in \mathbf{C},\left|z_{j}\right| \leq 1$, and $K+2 J=T$.

Proof. The construction of $B(\xi)$ utilizes an auxiliary polynomial $P_{A}(z)$, defined by

$$
P_{A}(z)=\frac{1}{2} \sum_{n=0}^{T-1} a_{T-n} z^{n}+a_{0} z^{T}+\frac{1}{2} \sum_{n=1}^{T} a_{n} z^{T+n}
$$

We note that

$$
P_{A}\left(e^{i \xi}\right)=e^{i T \xi} A(\xi),
$$

and hence $P_{A}$ is, up to a phase shift, an extension of $A$ from the unit circle to the complex plane. Now $Q_{A}(z)=z^{2 T} P_{A}\left(z^{-1}\right)$ is also a polynomial of degree $2 T$ on $\mathbf{C}$, and we see that

$$
Q_{A}\left(e^{i \xi}\right)=e^{2 i T \xi} P_{A}\left(e^{i \xi}\right)=e^{i T \xi} A(\xi) .
$$

Thus, $P_{A}(z)$ and $Q_{A}(z)$ are polynomials of degree $2 T$ which agree on the restriction to the unit circle, and hence must coincide. It follows that if $z_{0}$ is 
a root of $P_{A}(\alpha)$, then $z_{0}^{-1}$ is also a root. This implies that 0 is not a root, for instance. Moreover, since $\overline{P_{A}(z)}=P_{A}(\bar{z})$, we see that the nonreal roots of $P_{A}(z)$ must occur in conjugate pairs. Finally, if $z_{0}$ is a root of $P_{A}(z)$ on the unit circle, then, the multiplicity of this root is of even order, as a positive perturbation of $a_{0}$ to $a_{0}+\varepsilon$ will yield $P_{A_{\varepsilon}}(z)$ with no roots on the unit circle, and the conjugate pair $\left(z_{0}, \bar{z}_{0}\right)$ of roots will bifurcate into one or more quadruples of the form $\left(z_{\varepsilon}, z_{\varepsilon}^{-1}, \bar{z}_{\varepsilon}, \bar{z}_{\varepsilon}^{-1}\right)$ as $\bar{z}_{0}=z_{0}^{-1}$ on the unit circle.

Let $\left\{r_{1}, \ldots, r_{k}\right\}$ be the real roots of $P_{A}(z)$ of modulus less than one, and let $\left\{z_{1}, \ldots, z_{J}, \bar{z}_{1}, \ldots, \bar{z}_{J}\right\}$ be a choice of the nonreal roots of modulus less than one (counting multiplicities), and let $\left\{w_{1}, \ldots, w_{L}, \bar{w}_{1}, \ldots, \bar{w}_{L}\right\}$ be the roots of order two on the unit circle. So we must have $\left\{z_{1}, \ldots, z_{J}, \bar{z}_{1}, \ldots, \bar{z}_{J}, r_{1}\right.$, $\left.\ldots, r_{K}, w_{1}, \ldots, w_{L}, \bar{w}_{1}, \ldots, \bar{w}_{L}\right\}$ are $2 J+K+2 L=T$ roots of modulus less than or equal to one. There is an ambiguity in the choice of $\left\{z_{1}, \ldots, z_{J}\right\}$, and $\left\{w_{1}, \ldots, w_{L}\right\}$ but the real numbers $\left\{\operatorname{Re} z_{j},\left|z_{j}\right|^{2}\right\}$ and $\left\{\operatorname{Re} w_{j},\left|w_{j}\right|^{2}\right\}$ are unambiguously defined up to order. Define

$$
\begin{aligned}
B(\xi)= & {\left[\frac{1}{2}\left|a_{T}\right| \prod_{k=1}^{K}\left|r_{k}\right|^{-1} \prod_{j=1}^{J}\left|z_{j}\right|^{-2}\right]^{1 / 2} \cdot \prod_{k=1}^{K}\left(e^{i \xi}-r_{k}\right) \prod_{j=1}^{J}\left(e^{2 i \xi}-2 e^{i \xi} \operatorname{Re} z_{j}+\left|z_{j}\right|^{2}\right) } \\
& \cdot \prod_{l=1}^{L}\left(e^{2 i \xi}-2 e^{i \xi} \operatorname{Re} w_{j}+\left|w_{j}\right|^{2}\right),
\end{aligned}
$$

which is a trigonometric polynomial of order $T$ with real coefficients, and the coefficients of $B(\xi)$ are uniquely defined in terms of the choice of zeros of $P_{A}(\xi)$ made above.

We now show that $|B(\xi)|^{2}=A(\xi)$. First, we note that $P_{A}(z)$ can be expressed in terms of the roots as

$$
\begin{aligned}
P_{A}(z)= & \frac{1}{2} a_{T}\left[\prod_{k=1}^{K}\left(z-r_{k}\right)\left(z-r_{k}^{-1}\right) \cdot \prod_{J=1}^{J}\left(z-z_{j}\right)\left(z-\bar{z}_{j}\right)\left(z-z_{j}^{-1}\right)\left(z-\bar{z}_{j}^{-1}\right)\right] \\
& \cdot \prod_{l=1}^{L}\left(z-w_{j}\right)^{2}\left(z-\bar{w}_{j}\right)^{2} .
\end{aligned}
$$

Letting $z=e^{i \xi}$, and letting $z_{0}$ be any zero above, we see that

$$
\left|\left(e^{i \xi}-z_{0}\right)\left(e^{i \xi}-\bar{z}_{0}^{-1}\right)\right|=\left|z_{0}\right|^{-1}\left|e^{i \xi}-z_{0}\right|^{2}
$$

(noting for the case of $w_{j}$ that $\bar{w}_{j}^{-1}=w_{j}$ ). Now we note that since $A$ is nonnegative we have $A(\xi)=|A(\xi)|$. Hence

$$
\begin{aligned}
A(\xi)= & |A(\xi)|=\left|P_{A}\left(e^{i \xi}\right)\right| \\
= & {\left[\frac{1}{2}\left|a_{N}\right| \prod_{k=1}^{K}\left|r_{k}\right|^{-1} \prod_{j=1}^{J}\left|z_{j}\right|^{-2}\right] \cdot\left|\prod_{k=1}^{K}\left(e^{i \xi}-r_{k}\right) \prod_{j=1}^{J}\left(e^{i \xi}-z_{j}\right)\left(e^{i \xi}-\bar{z}_{j}\right)\right| } \\
& \cdot \prod_{J=1}^{L}\left(e^{i \xi}-w_{j}\right)\left(e^{i \xi}-\bar{w}_{j}\right) \\
= & |B(\xi)|^{2},
\end{aligned}
$$

where $B(\xi)$ is given above. 
Continuing with the proof of Theorem 3.2 we see that the mapping $\pi: \mathscr{M}_{N} \rightarrow$ $\mathscr{R}_{N}$ is surjective, since each point $b=\left(b_{1}, \ldots, b_{D}\right)$ of $\mathscr{R}_{N}$ yields a nonnegative polynomial

$$
A(\xi)=1+\sin \left(\frac{\xi}{2}\right)\left(\sum_{j=1}^{D} b_{j}\left(\frac{\cos \xi}{2}\right)^{2 j-1}\right),
$$

as the constraints which define $\mathscr{R}_{N}$ give the nonnegativity of the cosine polynomial $A(\xi)$ necessary to apply Lemma 3.3. Moreover, Lemma 3.3 yields a unique branch of the multisheeted mapping $\Pi: \mathscr{M}_{N} \rightarrow \mathscr{R}_{N}$, and we denote this branch by $\mathscr{M}_{N}^{0} \subset \mathscr{M}_{N}$, and we see that $\pi: \mathscr{M}_{N}^{0} \rightarrow \mathscr{R}_{N}$ is a one-to-one mapping.

We now want to extend this to obtain subsets of $\mathscr{R}_{N}$ which have specific analytic properties. Regularity of the scaling function associated with $a \in \mathscr{M}_{M}$ is determined by the maximum value of the magnitude of the trigonometric polynomial $\left|Q_{0}(\xi)\right|$ in the factorization $H(\xi)=\left(\left(1+e^{i \xi}\right) / 2\right) Q_{0}(\xi)$. More generally we will consider higher order factorizations. We will look at this in more detail in the next section.

\section{Moment-Free Wavelet Systems}

We now want to investigate the parametrization of wavelets with specified vanishing moments. All wavelet systems have vanishing zeroth moment, for the wavelet function $\psi(x)$, corresponding to $h \in M_{N}$, we have $\int_{\mathbf{R}} \psi(x) d x=$ 0 , and this corresponds to $\hat{\psi}(0)=0$. We shall say that a wavelet system $\{\varphi(x), \psi(x)\}$ is moment-free of order $M$ if

$$
\int_{\mathbf{R}} x^{j} \psi(x) d x=0, \quad j=0, \ldots, M .
$$

Lemma 4.1. A wavelet system $\Psi$, corresponding to $a \in M_{N}$ is moment-free of order $M$ if and only if

$$
H(\xi)=\left(\left(1+e^{i \xi}\right) / 2\right)^{M+1} Q(\xi),
$$

for some trigonometric polynomial

$$
Q(\xi)=\sum_{k=0}^{N-M-2} c_{k} e^{i k \xi}
$$

Proof. Let $\psi(x)$ be the wavelet function in the wavelet system $\Psi$. Then

$$
\int_{\mathbf{R}} x^{j} \psi(x) d x=0, \quad j=0, \ldots, M,
$$

if and only if $\hat{\psi}(\xi)$ vanishes to order $M$ at $\xi=0$. Now we have defined

$$
\psi(x)=\sum_{k=-1}^{N-2} h_{k+1}(-1)^{k} \varphi(2 x+k),
$$

and it follows that

$$
\hat{\psi}(\xi)=H \overline{\left(\frac{1}{2} \xi+\pi\right)} \hat{\varphi}\left(\frac{1}{2} \xi\right)
$$


Moreover, from the factorization (4.1) $H(\xi)$ has a zero of order $M+1$ at $\xi=\pi$, and by $(4.2)$ we see that $\hat{\psi}(\xi)$ vanishes to order $(M+1)$ precisely when (4.1) is valid. This proves the lemma.

The parametrization space for all wavelet systems of order $N$ is a compact submanifold of $\mathbf{R}^{N}$ with some singularities, all of generic dimension $D=$ $(N-1) / 2$, as we have seen before. The condition (4.1) imposes $M$ additional linear condition on the coordinates $\left(a_{0}, \ldots, a_{N-1}\right)$ of a point $a \in M_{N}$. Let us denote these conditions by affine functions $L_{j}=\mathbf{R}^{N} \rightarrow \mathbf{R}$, so that $\bigcap_{j=1}^{M} L_{j}^{-1}(0) \cap$ $\mathscr{M}_{N}$ is precisely the set of all wavelet systems which are moment-free of order $M$. Generically, we can have no more than $D$ additional independent linear constraints, and if we let $\mathscr{M}_{N, M}$ be the wavelet systems of order $N$ which are moment-free of order $M$, then we see that we should have

$$
\operatorname{dim} \mathscr{M}_{N, M}=D-M, \quad M=0,1, \ldots, D .
$$

Let $\mathscr{R}_{N, M}$ denote the image of $M_{N, M}$ under the mapping $\pi: \mathscr{M}_{N} \rightarrow \mathscr{R}_{N}$.

Theorem 4.2. $\mathscr{R}_{N, M}$ is an affine submanifold of $\mathscr{R}_{N}$ of codimension $M$, and $\mathscr{M}_{N, M}^{0}$ is a submanifold of $\mathscr{M}_{N}^{0}$ of codimension $M$.

Thus we see that the moment-free conditions are nontrivial for wavelet systems for which they make sense. Since the factorization (4.1) has a maximal factor of order $D+1$ we see that the wavelet system cannot have higher vanishing moments.

We shall first show that for $M<D, \mathscr{R}_{N, M}$ is the closure of an open set $\mathscr{R}_{N, M}^{0} \subset \mathbf{R}^{D-M}$, and there are natural containments $\mathscr{R}_{N, 0}^{0} \supset \mathscr{R}_{N, 1}^{0} \supset \cdots \supset$ $\mathscr{R}_{N, D-1}^{0}$, and that $\mathscr{R}_{N, D}$ is a point, the Daubechies point, satisfying $D_{N}:=$ $\mathscr{R}_{N, D} \in \mathscr{R}_{N, D-1}^{0}$. We shall see that there are affine hyperplanes $H_{j}$ in $\mathbf{R}^{D}$ such that $\mathscr{R}_{N, M}=\mathscr{R}_{N, 0} \cap H_{1} \cap \cdots \cap H_{M}$. We will parametrize each $\mathscr{R}_{N, M}$ as an open subset of $\mathbf{R}^{D-M}$ and then embed it in an affine function into $\mathscr{R}_{N, M-1}$. First we define the Daubechies point $D_{M}$.

Consider a maximal factorization of the form

$$
H(\xi)=\left(\left(1+e^{i \xi}\right) / 2\right)^{1+D} Q_{D}(\xi),
$$

then in this case, we must have, by Theorem 3.1

$$
\left|Q_{D}(\xi)\right|^{2}=P_{D}\left(\sin ^{2}\left(\frac{\xi}{2}\right)\right)+0,
$$

as there are no degrees of freedom left.

Reexpressing $Q_{D}(\xi)$ in terms of the parameters of $R_{N, 0}$ we see that

$$
\left|Q_{D}(\xi)\right|^{2}=1+\sum_{k=1}^{D}\left(\begin{array}{c}
D+k \\
h
\end{array}\right) \sin ^{2 k}\left(\frac{\xi}{2}\right),
$$

and since

$$
\begin{aligned}
|H(\xi)|^{2} & =\left(\frac{1+e^{i \xi}}{2}\right)^{2}\left|Q_{0}(\xi)\right|^{2}=\left(\frac{1+e^{i \xi}}{2}\right)^{2+2 D}\left|Q_{D}(\xi)\right|^{2} \\
& =\left[2 \cos ^{2}\left(\frac{\xi}{2}\right)\right]^{2 D}\left|Q_{D}(\xi)\right|^{2}=[(1+\cos \xi)]^{D}\left|Q_{D}(\xi)\right|^{2}
\end{aligned}
$$


Thus we have the equality

$$
\begin{gathered}
(1+\cos \xi)^{D}\left(1+\sum_{k=1}^{D}\left(\begin{array}{c}
D+k \\
k
\end{array}\right) \sin ^{2 k}\left(\frac{\xi}{2}\right)\right) \\
=1+\sin ^{2 D+2}\left(\frac{\xi}{2}\right) \sum_{j=1}^{D} b_{j}(\cos \xi)^{2 j-1} .
\end{gathered}
$$

By expressing both sides as polynomials in $\cos \xi$, and comparing coefficients we get explicit values for $\left(b_{1}, \ldots, b_{D}\right)$ corresponding to this choice of $\left|Q_{0}(\xi)\right|^{2}$. Since $\left|Q_{D}(\xi)\right|^{2}>1$ for all $\xi$, we see that $\left(b_{1}, \ldots, b_{D}\right)$ in this case in an interior point of $\mathscr{R}_{N, 0}$. This determines the coordinates of $D_{N}$ as a point of $\mathscr{R}_{N}$, and since $\left|Q_{D}(\xi)\right|^{2} \geq 1$ for all $\xi \in \mathbf{R}$, we see that $D_{N}$ is an interior point of $\mathscr{R}_{N}$ (and for the same reason will turn out to be an interior point of $\mathscr{R}_{N, M}$ for $M=1, \ldots, D-1$, or we will see. We will see examples of these calculations in the next section.

Suppose $H(\xi)$ is a wavelet system moment-free of order $M$, then

$$
H(\xi)=\left(\left(1+e^{i \xi}\right) / 2\right)^{M+1} Q_{M}(\xi),
$$

for specific coefficients of the trigonometric polynomial $Q_{M}(\xi)$, and hence $\left|Q_{M}(\xi)\right|^{2}$ is a well-defined cosine polynomial, as before. Then we have

$$
\left|Q_{M}(\xi)\right|^{2}=P_{M}\left(\sin ^{2}\left(\frac{\xi}{2}\right)\right)+\sin ^{2 M+2}\left(\frac{\xi}{2}\right) \sum_{J=1}^{D-M} \tilde{b}_{j}(\cos \xi)^{j-1} .
$$

Now again we have

$$
(1+\cos \xi)^{D-M}\left|Q_{M}(\xi)\right|^{2}=\left|Q_{0}(\xi)\right|^{2}=1+\sin ^{2}\left(\frac{\xi}{2}\right) \sum_{j=1}^{D} b_{j}(\cos \xi)^{j},
$$

Thus the $b_{j}$ are linearly related to the $\tilde{b}_{j}$, and we can solve for $b_{1}, \ldots, b_{b}$ as affine functions of $\tilde{b}_{1}, \ldots, \tilde{b}_{D-M}$. This is the required affine embedding.

\section{EXAMPLES}

In this section we want to explicitly describe various aspects of the reduced moduli space for wavelet systems introduced in $\S 3$. Let us recall that for $N \in$ $2 \mathrm{Z}^{+}$, we defined, for $N \geq 4$,

$$
\mathscr{R}_{N}=\left\{\left(b_{1}, \ldots, b_{D}\right) \in \mathbf{R}^{D}: 1+y \sum_{j=1}^{D} b_{j}\left(\frac{1}{2}-y\right)^{2 j-1} \geq 0\right\},
$$

where $D=(N-2) / 2$. We note that each $\mathscr{R}_{N}$ is a closed convex subset of $\mathbf{R}^{D}$, since $\mathscr{R}_{N}$ is the intersection of closed half spaces in $\mathbf{R}^{D}$. Moreover, $\mathscr{R}_{N}$ is compact since it is the image of the compact set $\mathscr{M}_{N}$ under the continuous mapping given by Theorem 3.2.

Let us look at $\mathscr{R}_{4}$ first to get a better idea of these constraints. We have

$$
\mathscr{R}_{4}=\left\{b_{1} \in \mathbf{R}: 1+b_{1} y\left(\frac{1}{2}-y\right) \geq 0 \text {, for all } y \in[0,1]\right\},
$$

and we can write this in the form

$$
\mathscr{R}_{4}=\left\{b_{1}: b_{1} g_{1}(y) \geq-1,0 \leq y \leq 1\right\},
$$


for $g_{1}(y)=y\left(\frac{1}{2}-y\right)$. We then set

$$
g_{1 M}=\max _{y \in[0,1]} g_{1}(y), \quad g_{1 m}=\min _{y \in[0,1]} g_{1}(y) .
$$

Now we shall see explicitly that $g_{1 M}>0$ and $g_{1 m}<0$, and then we obtain

$$
b_{1} g_{1}(y) \geq-1, \quad 0 \leq y \leq 1,
$$

implies that $b_{1} g_{1 M} \geq-1$ which implies that $b_{1} \geq-1 / g_{1 M}$. Similarly, we see

$$
b_{1} g_{1}(y) \geq-1, \quad 0 \leq y \leq 1,
$$

implies that $b_{1} g_{1 m} \geq-1$, which implies, since $g_{1 m}<0$, that $b_{1} \leq-1 / g_{1 m}$. Thus we obtain $-1 / g_{1 M} \leq b_{1} \leq-1 / g_{1 m}$. Now by elementary calculus we find that $g_{1}$ has a single critical point at $y=\frac{1}{4}$, and has a maximum value with $g_{1}\left(\frac{1}{4}\right)=\frac{1}{16}$. Similarly, we see that $g_{1 m}=g_{1}(1)=-\frac{1}{2}$. Then we obtain $-16 \leq b_{1} \leq 2$. Suppose that $b_{1}$ satisfies

$$
-1 / g_{1 M} \leq b_{1} \leq-1 / g_{1 m} .
$$

Now suppose that $b_{1} \geq 0$, then, for any $y \in[0,1]$,

$$
b_{1} g_{1}(y) \geq b_{1} g_{1 m} \geq-1,
$$

and similarly, if $b_{1}<0$, we have that, for $0 \leq y \leq 1$,

$$
b_{1} g_{1}(y) \geq b_{1} g_{1 M} \geq-1 \text {, }
$$

and hence we see that $\mathscr{R}_{4}=[-16,2]$.

These arguments extend somewhat to higher dimensions. Let $g_{j}(y)=$ $y\left(\frac{1}{2}-y\right)^{2 j-1}$. Then let

$$
g_{j M}=\max _{y \in[0,1]} g_{j}(y), \quad g_{j m}=\min _{y \in[0,1]} g_{j}(y),
$$

and we need to compute the max and min of $g_{j m}$ on $[0,1]$.

Lemma 5.1.

$$
\begin{aligned}
g_{j M} & =g_{j}\left(\frac{1}{4 j}\right)=\frac{1}{4 j}\left(\frac{1}{2}-\frac{1}{4 j}\right)^{2 j-1}>0, \\
g_{j m} & =g_{j}(1)=\left(-\frac{1}{2}\right)^{2 j-1}=-\frac{1}{2^{2 j-1}}<0 .
\end{aligned}
$$

Proof. There are only two critical points for $g_{j}$ on $\mathbf{R}$, namely at $y=1 / 4 j$ and at $y=\frac{1}{2}$. The latter has multiplicity $2 j-2$, and since $g_{j}\left(\frac{1}{2}\right)=0$, and $g_{j}(1)$ is negative, it follows that $g_{j}(1)$ must be the minimum on $[0,1]$, and that the maximum of $g_{j}$ on $[0,1]$ occurs at the local maximum at the critical point $y=1 / 4 j$. Thus the lemma is proved.

Theorem 5.2. The reduced moduli space $\mathscr{R}_{N}$ contains the polyhedron

$$
\Delta_{N}=\left\{\left(b_{1}, \ldots, b_{D}\right) \in \mathbf{R}^{D}: \sum_{j=1}^{D} b_{j} g_{j \varepsilon_{j}} \geq-1\right\}
$$

where, for $J=1, \ldots, D$,

$$
g_{j 0}:=\max _{y \in[0,1]} g_{j}(y), \quad g_{j 1}:=\min _{y \in[0,1]} g_{j}(y),
$$


and $\varepsilon_{j} \in\{0,1\}$, yielding $2^{D}$ distinct linear inequalities. Moreover, the vertices of $\Delta_{N}$ are points on the boundary of the reduced moduli space $\mathscr{R}_{N}$, and they have the form

$$
\begin{gathered}
\left(g_{10}^{-1},-g_{11}^{-1}, 0, \ldots, 0\right), \\
\left(0,0, g_{20}^{-1},-g_{21}^{-1}, 0, \ldots, 0\right), \\
\vdots \\
\left(0,0, \ldots, 0, g_{D 0}^{-1},-g_{D 1}^{-1}\right) .
\end{gathered}
$$

For instance $\Delta_{6}$ is given by

$$
\begin{aligned}
\Delta_{6}=\left\{\left(b_{1}, b_{2}\right) \in \mathbf{R}^{2}:\right. & -\frac{1}{2} b_{1}-\frac{1}{8} b_{2} \geq-1, \frac{1}{16} b_{1}-\frac{1}{8} b_{2} \geq-1, \\
& \left.-\frac{1}{2} b_{1}+\frac{3^{3}}{2^{12}} b_{2} \geq-1, \frac{1}{16} b_{1}+\frac{3^{3}}{2^{12}} b_{2} \geq-1\right\} .
\end{aligned}
$$

Let us calculate where the Haar point and Daubechies points are in this example of $\mathscr{R}_{6}$. Recall that $D_{N}$ is a point of $\mathscr{R}_{N}$ defined by setting

$$
Q_{0}(\xi)=\left(\left(1+e^{i \xi}\right) / 2\right)^{D} Q_{D}(\xi),
$$

where $\left|Q_{D}\right|^{2}$ is explicitly given by

$$
\left|Q_{D}(\xi)\right|^{2}=P_{D}\left(\sin ^{2}\left(\frac{\xi}{2}\right)\right)=\sum_{k=0}^{D}\left(\begin{array}{c}
D+k \\
k
\end{array}\right) \sin ^{2 k}\left(\frac{\xi}{2}\right) .
$$

Let $N=4$, and we see that $D=1$, and

$$
H(\xi)=\left(\left(1+e^{i \xi}\right) / 2\right)^{2} Q_{1}(\xi)
$$

where

$$
\left|Q_{1}(\xi)\right|^{2}=1+2 \sin ^{2}\left(\frac{\xi}{2}\right)=2-\cos \xi
$$

and at the same time

$$
\left(\left(1+e^{i \xi}\right) / 2\right) Q_{1}(\xi)=Q_{0}(\xi),
$$

where

$$
\begin{aligned}
\left|Q_{0}(\xi)\right|^{2} & =1+\sin ^{2}(\xi / 2) \cdot b_{1} \cdot \cos \xi \\
& =1+b_{1}((1-\cos \xi) / 2) \cos \xi \\
& =1+\left(b_{1} / 2\right) \cos \xi-\left(b_{1} / 2\right) \cos ^{2} \xi .
\end{aligned}
$$

From (5.2) we find that

$$
\left|\left(\left(1+e^{i \xi}\right) / 2\right)\right|^{2}\left|Q_{1}(\xi)\right|^{2}=\left|Q_{0}(\xi)\right|^{2}
$$

which implies that, using (5.1)

$$
((1-\cos \xi) / 2)(2-\cos \xi)=1+\left(b_{1} / 2\right) \cos \xi-\left(b_{1} / 2\right) \cos ^{2} \xi
$$

which holds if and only if $b_{1}=1$. Moreover, we have that if $b_{1}=0$, we have

$$
H(\xi)=\left(\left(1+e^{i \xi}\right) / 2\right) \cdot 1
$$


which is the trigonometric polynomial corresponding to the Haar point $\left(a_{0}, a_{1}\right)$ $=(1,1)$, or $\left(h_{0}, h_{1}\right)=\left(\frac{1}{2}, \frac{1}{2}\right)$. Thus we see that $H=(0,0)$ is the origin in this rhombus, while $D_{4}$ is the point $(1,0)$. By a calculation similar to the one above, it is easy to check that in the affine 2-plane

$$
D_{6}=D_{4}+\left(\frac{3}{4},-\frac{3}{4}\right)=\left(\frac{7}{4},-\frac{3}{4}\right) \text {. }
$$

The moduli space of moment-free wavelet systems of order 1 is the affine line passing through the two points $D_{4}$ and $D_{6}$.

The proof of Theorem 5.2 is omitted as it is a straightforward adoption of the one-dimensional case, and consists of verifying sequentially a sequence of inequalities in both directions, when restricted to the coordinate axes.

The boundary component of $\mathscr{R}_{N}$ are algebraic surface of degree $\geq 1$, although it seems that at least one of the faces of $\Delta_{N}$ coincides with a portion of the boundary of $\mathscr{R}_{N}$. A computer description of $\mathscr{R}_{N}$ has been carried out, and wavelet systems which vary as points of the reduced moduli space have been coded, and optimization studies have been initiated. This will be reported on more fully in subsequent publications.

\section{REFERENCES}

1. Ingrid Daubechies, Orthonormal bases of compactly supported wavelets, Comm. Pure. Appl. Math. 41 (1988), 906-996.

2. A. Haar, Math. Ann. 69 (1910), 336.

3. W. Lawton, Tight frames of affinely supported wavelets, J. Math. Phys. 31 (1990), 19881901.

4. D. Pollen, Parametrization of compactly supported wavelets, Company Report, Aware, Inc., AD890503.1.4, May, 1989.

5. G. Polya and G. Szegö, Aufgaben und Lehrsätze aus der Analysis, Vol. II, Springer, Berlin, 1971.

Department of Mathematics, Rice University, Houston, Texas 77251

E-mail address: wells@rice.edu 